\title{
ACOGIMIENTO FAMILIAR: ESTUDIO DE LAS INTERACCIONES ANTE LAS VISITAS FAMILIARES
}

\section{JOSÉ RAMÓN BUENO ABAD}

Catedrático de Psicología Social. Universitat de València.

JOSÉ VICENTE PÉREZ COSÍN

Profesor Asociado de Trabajo Social E.U. Trabajo Social. Universitat de València.

\section{CLAVES CONCEPTUALES}

Desamparo, protección, tutela, acogimiento familiar, régimen de visitas, retorno, adopción.

Interacción padres e hijos, emociones, reacciones físicas, socialización, integración social.

\section{ABSTRACT}

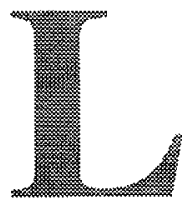

a ley orgánica 1/96 sobre Protección Jurídica del niño, establece una medida de protección denominada acogimiento familiar preadoptivo, en aquellos supuestos en donde se valora como irreversible el retorno a la familia de origen del niño, y en donde se dispone de una familia alternativa para la integración social de los niños.

La regulación del régimen de visitas compete, en primer lugar, a la administración responsable de la protección a la infancia y, en segundo lugar, a la Fiscalía de Menores, quien a través del Juzgado de Familia podrá regular judicialmente el régimen de visitas.

El desarrollo de estas visitas y su plasmación en actos concretos está mediatizado por la complejidad de la situación y por las circunstancias relacionadas con la estabilidad emocional del niño, consecuencia del desequilibrio producido por la desestructuración familiar anterior.

La no-justificación del régimen de visitas puede plantearse cuando existen problemas emocionales que hacen tambalear el equilibrio del acogimiento familiar y, sobre todo, en los casos en que está en juego la integración social del niño, a través de su familia acogedora. 


\section{INTRODUCCIÓN}

En los servicios de protección a la infancia, se desarrollan, entre otros, los programas de Acogimiento Familiar como medidas adecuadas para la integración social de los/as niños/as en situación de desamparo.

La ley orgánica 1/96 sobre Protección Jurídica del niño, establece una medida de protección, denominada acogimiento familiar preadoptivo, en aquellos supuestos en donde se valora como irreversible el retorno a la familia de origen del niño, y en donde se dispone de una familia alternativa para la integración social de los niños.

Además de las particularidades anteriores, este tipo de medida de protección tiene otras características reguladas por la legislación civil, que implican un proceso por medio del cual la familia de origen conoce la situación del niño, materializándose de hecho en un régimen de visitas.

La regulación del régimen de visitas compete, en primer lugar, a la administración responsable de la protección a la infancia, y en segundo lugar, a la Fiscalía de Menores quien a través del Juzgado de Familia podrá regular judicialmente el régimen de visitas.

El proceso de asignación de las visitas familiares se inicia a instancia de los progenitores o del niño o niña, si es menor de 12 años, como mencionamos en el párrafo anterior, bien en la entidad pública que ejerce la tutela del niño/a, o a través del juzgado de familia correspondiente, previo informe del fiscal de menores que es la figura institucional de superior vigilancia en la protección de los niños/as tutelados/as.

Recibida la petición, se estudia la idoneidad del régimen de visitas. Los profesionales que atienden al niño y los profesionales de los servicios sociales públicos que conocen a los progenitores la valoran conjuntamente, emitiendo informe positivo, pautando: temporalidad, frecuencia y número de personas autorizadas a realizar las visitas.

El interés fundamental, en este proceso, es el del niño, y la finalidad última es la integración social con las máximas garantías de bienestar.

En el caso de ser asignado un régimen de visitas por el Juzgado de Familia, corresponde al equipo psicosocial del mismo emitir informe favorable o desfavorable comunicándolo a la entidad pública responsable de la tutela del niño/a.

El desarrollo de estas visitas, y su plasmación en actos concretos, está mediatizado por la complejidad de la situación y por las circunstancias relacionadas con la estabilidad emocional del niño, consecuen- 
cia del desequilibrio producido por la desestructuración familiar anterior.

La no-justificación del régimen de visitas puede plantearse cuando existen problemas emocionales que hacen tambalear el equilibrio del acogimiento familiar y, sobre todo, en los casos en que está en juego la integración social del niño, a través de su familia acogedora.

Desde esta perspectiva, se plantea este estudio con la intención de contribuir a la mejora de la calidad de los servicios de protección a la infancia, y con la finalidad de mejorar la eficacia de una medida de protección como es el Acogimiento Familiar.

\section{MARCO TEÓRICO}

El trabajo que presentamos responde a la necesidad de profundizar en las distintas incidencias que se producen en el ámbito del acogimiento familiar. En este sentido, nos ha interesado profundizar en el conocimiento de las situaciones de interacción entre los niños en situación de acogimiento familiar y sus familias naturales.

Desde el punto de vista teórico, hemos de considerar este interés como una consecuencia de los mecanismos de creación del vínculo, tal y como históricamente fue recogido por Bowlby (1979): la Attachment Theory describe cómo los sujetos necesitados de protección necesitan disponer de un sistema de conducta relativamente estable que les facilite la posibilidad de vinculación. Estas conductas de vinculación son fundamentales para el desarrollo de los lazos afectivos y psicológicos de los niños en situación de crecimiento evolutivo. En este sentido, en las situaciones de desestructuración, abandono, malos tratos, dificultades, etc. , nos encontramos con una serie de rupturas en la creación del sistema vínculo, y para tratar de intervenir sobre estos mecanismos se plantean distintos instrumentos; entre ellos: el acogimiento familiar.

Como han recogido Ripol-Millet y Rubiol (1990), existen distintas perspectivas del acogimiento familiar para niños con problemas de adaptación, conducta, abandono, etc. Pero siempre este tipo de instrumentos pretenden ser una ayuda para que las familias que tienen dificultades temporales para hacerse cargo de alguno de sus miembros puedan beneficiarse eficazmente de la colaboración solidaria de otras personas o familias. Partiendo de estas características generales, y siendo coherentes con el sentido temporal de esta medida de acogimiento, hemos pretendido analizar las situaciones de interacción como una medida que se sitúa en la intervención psicoeducativa con el niño, tendente a mejorar y mantener sus relaciones de consolidación de los vínculos afectivos. 
El planteamiento de análisis de estas circunstancias surge de considerar estas medidas como parte de un mecanismo de apoyo social, tal como recogía históricamente Caplan (1974), como la creación de una serie de vínculos entre el niño y un grupo, en este caso la familia acogedora, para promover el dominio emocional, orientar en el comportamiento y aportar y estimular acerca de la propia identidad.

Desde esta perspectiva, hemos de destacar que los elementos de conformación de la identidad generan un desarrollo evolutivo, tal y como ha recogido L'Ecuyer (1990): la identidad evoluciona y varía según las distintas etapas, teniendo en consideración las primeras fases de emergencia del sí mismo ( 0 a 2 años), la confirmación del sî mismo (de 2 años a los 5 años) y la expansión de la identidad del sí mismo (de los 5 años a los 12 años), resultando decisivas las actuaciones e intervenciones psicosociales que se puedan plantear en dichas etapas.

Por último, hemos planteado este trabajo en el análisis de un proceso de interacción en un ámbito donde están presentes aspectos legales, sociales y psicológicos. Entendemos nuestro trabajo dentro de la definición de un espacio psicosocial. Para tratar de articular este proceso, consideramos que la intervención psicosocial tiene que tratar de enlazar los procesos de conexión entre lo psicológico y lo social, y en este sentido podemos plantear las posiciones de Doise (1982) para tratar de asentar la misma sobre cuatro niveles de explicación y de análisis.

- En primer lugar, el nivel de explicación intraindividual, por el cual el sujeto organiza sus percepciones y su experiencia del entorno social y los mecanismos cognitivos que llevan a esa percepción.

- Un segundo nivel reconocido como interindividual que pretende analizar lo que ocurre entre los individuos dentro de una situación, dada, qué dinámicas relacionales y organizacionales se desarrollan; se trata en este caso de un nivel de explicación intraindividual o intrasituacional.

- Un tercer nivel de explicación, el análisis posicional, esto es, las diferentes posiciones sociales entre los individuos y entre los grupos; se trata de precisar los efectos de estas diferencias, de status, de categorías, etc., sobre las interacciones que los individuos y los grupos mantienen entre ellos.

- Un cuarto tipo de trabajos que se fundamentan en una explicación ideológica, esto es, investigaciones que hacen intervenir las normas, las ideas, las creencias de un grupo social determinado y que buscan el análisis de las evidencias de sus funciones y efectos sobre las interacciones que se desarrollan entre los individuos y los grupos. 
Desde nuestra perspectiva, y para ser consecuentes con las propuestas psicosociales, tal como hemos recogido en Bueno Abad (1999), hemos optado por un análisis de la interacción desde el nivel interindividual. Esto es, la definición de la interacción desde una perspectiva ternaria generando una perspectiva tripolar: el niño, la familia natural y la familia acogedora.

Optamos por un análisis de las reacciones de los sujetos observados, en el que están en curso los procesos de identidad, las actitudes de las familias naturales, las expectativas de las familias acogedoras y las responsabilidades de la administración encargada de la intervención en el proceso. Los elementos de referencia de dicho proceso de interacción han sido dimensionados en función de variables emocionales medidas a través de las referencias a las teorías de activación-cognición-observación, siguiendo los planteamientos de Gergen (1982).

\section{METODOLOGÍA}

La metodología en un estudio de este tipo implica un proceso previo de conocimiento de las medidas de protección a la infancia en situación de desamparo, un proceso inicial de reflexión, para la determinación del problema que pretendemos investigar, y un proceso de elaboración, para plantear el objeto de observación, la formulación de la hipótesis causal que nos oriente en el planteamiento de las variables a observar en cada sesión, y que nos conduzca al diseño más idóneo, según el tipo de variables que debamos investigar.

\subsection{Determinación del problema}

En los procesos de Acogimiento familiar sin previsión de retorno se pueden producir cambios en la estabilidad emocional de los niños, manifestando conductas alteradas como consecuencia de los desequilibrios causados por las visitas a su familia de origen.

Cuando el pronóstico de la situación de los niños es de no retorno a su familia de origen, las conductas (reacciones emocionales) de los niños suelen dificultar el desarrollo material de los regímenes de visitas, además de producir situaciones tensas, en el lugar donde se producen las visitas. Generando, de hecho, una actividad contradictoria con las perspectivas y posibles alternativas de futuro.

De otro lado y para otro tipo de situaciones, las visitas familiares intentan mantener un clima de relación afectiva positiva entre el niño y su familia de origen; de manera que, tras la suspensión de la medida de protección, se pueda continuar con la convivencia familiar sin ocasio- 
nar problemas al niño al reintegrarlo a su familia de origen.

Parece que, en este sentido y para este tipo de situaciones, se muestra la coherencia e idoneidad de este tipo de medidas.

\subsection{Hipótesis}

La hipótesis que nos orienta en este estudio se podría resumir en: «Las visitas familiares de los niños en Acogimiento familiar provocan conductas (reacciones emocionales) en ellos, que suponen situaciones contradictorias y de falta de armonía, lo que podría justificar, para algunas situaciones y casos concretos, la suspensión de las visitas, en interés del niño, que es prevalente al de su familia».

\section{SUJETOS}

Los sujetos a observar son la totalidad de los niños que están en acogida familiar en la provincia de Valencia, durante el primer semestre del año 1995.

La referencia concreta de los niños es la siguiente: nos encontramos con un grupo de niños (ocho) comprendidos entre uno y seis años, que identificaremos por siglas (P.R. 3 años, R.L. 4 años, A.L. 4 años, V.A. 2 años, J.M.A. 2 años, C.L. 6 años, C.M. 2 años y A. G. 1 año), según muestra el gráfico 1.

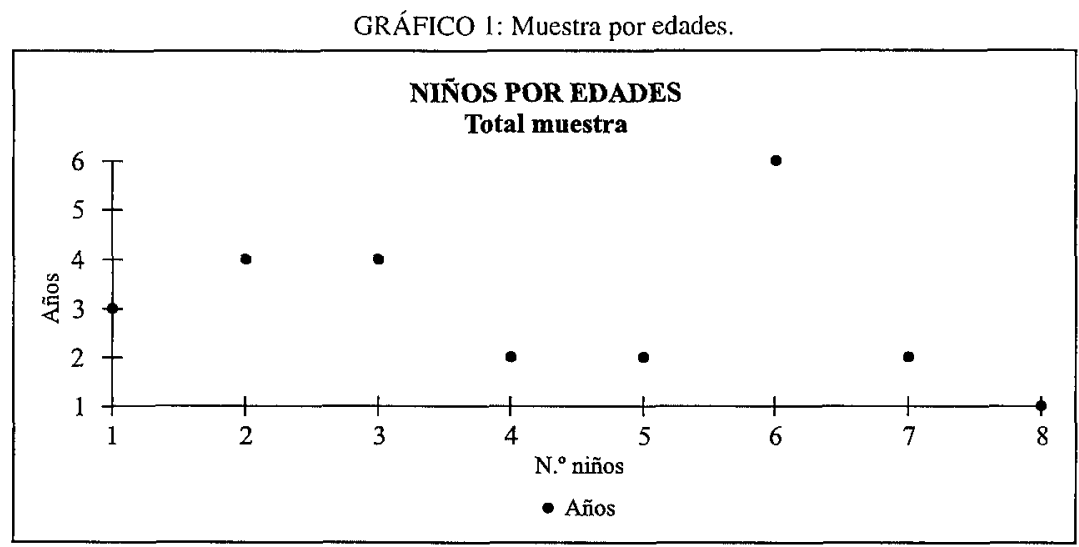

Elaboración propia. 


\section{VARIABLES}

Las conductas que presentan los niños se dimensionan en reacciones de base física y en reacciones emocionales consecuencia de la situación objeto de estudio (visitas), pudiéndose categorizar las variables para la observación (Anguera, 1992: 203-204) en:

a) Categorías de base física:

1- llanto,

2 - rostro frío,

3 - sonrisa.

b) Categorías de conductas consecuencia de las visitas:

4- tristeza,

5- alegría,

6 - indiferencia.

En este sentido, la elección de las variables investigadas ha venido justificada por la presencia de una interacción interindividual, tal como recoge Doise (1982) cuando plantea el estudio de una situación dada ante las dinámicas relacionales que se plantean en las mismas. En este estudio, ambas categorías están relacionadas, de tal forma que la observación de una conducta (b), pueda ser validada por la medición de una reacción física (a). Desde esta perspectiva, existe una correspondencia directa entre ambas categorías, expresada en la tabla 1:

TABLA 1: Correspondencia entre categorías.

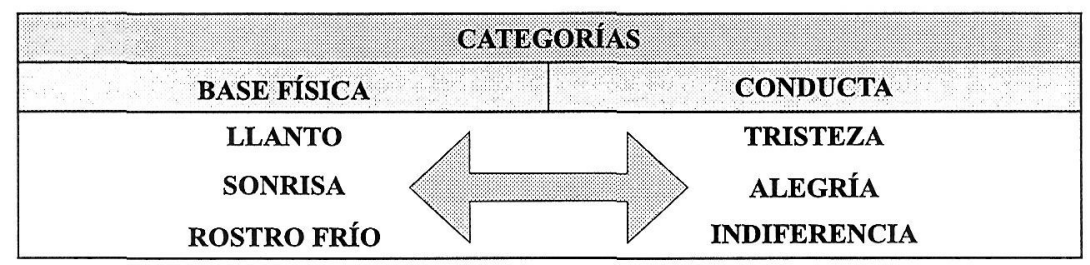

También es necesario indicar que las categorías son la expresión de los sentimientos de los niños ante el hecho (visitas de sus padres biológicos) objeto de estudio y que, por lo tanto, se entiende que expresan conductas favorables o positivas y conductas desfavorables o negativas, valorando que existe también una correspondencia entre las categorías y la conducta ante las visitas, según se presenta en la tabla 2 en la página siguiente. 
TABLA 2: Tipos conductas ante las visitas.

\begin{tabular}{|c|c|}
\hline & TIPO DE CONDUCTA \\
\hline FAVORABLE (positiva) & DESFAVORABLE (negativa) \\
\hline Sonrisa & Llanto \\
\hline Alegría & Tristeza \\
\hline \multirow{2}{*}{} & Rostro Frio \\
\hline & Indiferencia \\
\cline { 2 - 2 }
\end{tabular}

\section{DISEÑO}

En este apartado seguiremos a Anguera (1992: pp 33-69) y a Clemente (1992: pp 224-245). En primer lugar, una vez determinada la categorización de las conductas que deberemos observar, queda por definir cómo vamos a realizar el control de fiabilidad de la observación, por lo que optamos por realizar la determinación de fiabilidad interjueces y, posteriormente, calcular el índice de concordancia.

En segundo lugar, el nivel de descripción de las variables será molar, ya que las variables, aunque algunas de ellas son de base física, prevalece una descripción por las consecuencias que producen en los niños las visitas familiares.

Una vez determinadas las conductas que son útiles a nuestro problema hemos sistematizado las medidas que pretendemos obtener y la forma de registrar las categorías. La dimensión temporal es importante, ya que la forma que adopta la conducta de los niños es un estado duradero, en el que interesa apreciar el comienzo y el final de las conductas.

El tipo de medida que nos interesa observar es la duración de las conductas de los niños en las categorías definidas como unidades de conducta, aunque no debemos descartar tanto la frecuencia como la intensidad de determinadas unidades, ya que, dado el número de sesiones (24), pueden aportar algunas medidas significativas por frecuencia e intensidad.

Y en tercer lugar, la estrategia que utilizamos para la observación es la de un muestreo focal, delimitando los focos de observación a nivel individual, es decir, cada niño/a será un foco, y la estrategia de registro será un registro continuo de las conductas de todos los niños con especial interés en los periodos focales, que se definen en tres de cinco minutos de duración, apoyándonos en una estrategia tipo scan, para el grupo de niños en Acogimiento familiar, al inicio y al final de cada sesión. 


\section{PROCEDIMIENTO DE LA INVESTIGACIÓN}

Definir el procedimiento implica pautar el proceso de investigación observacional, partiendo de que es necesario contar en este caso con un conocimiento previo de lo que significa un régimen de visitas, qué regulación tiene a nivel judicial y qué repercusiones conductuales produce en las partes (padres e hijos).

\subsection{Lugar}

El lugar en donde se desarrollan las visitas es un centro residencial de protección, dependiente de la Dirección General de Servicios Sociales de la Generalidad Valenciana, ubicado en un municipio cercano a la capital de la provincia.

Este centro reúne unas condiciones especiales para el desarrollo de las sesiones: cuenta con puertas de entrada diferentes para las familias y para los niños que acuden acompañados por sus acogedores, además de contar con una sala especial para el desarrollo de la visita con un patio anexo para el juego, y en todo momento la relación niño/familia está supervisada por los educadores encargados de esta función.

\subsection{Sesiones}

Las sesiones se realizan semanalmente, en día sábado, en horario de 10 a 12 de la mañana, de forma que se realicen 4 sesiones al mes. Las sesiones se programan con antelación, y se comunican por escrito a las partes.

El total de sesiones de observación será de 24 , en cada una de las cuales cada niño ha sido observado como mínimo en 6 ocasiones, lo que nos permite contar con 144 registros de observación para cada niño observado.

\subsection{Desarrollo de la observación}

El momento de inicio de la sesión es cuando el niño es recibido por algún educador y se le acompaña a la sala de visita, teniendo el primer periodo focal de observación, para cada foco, puede ser secuenciado ya que los niños no acuden a la vez ni tienen por qué salir juntos; a continuación, se realizará un scan del grupo, una vez estén todo los niños en la sala. A la mitad de la visita, es decir, cuando ha transcurrido una hora, viene otro periodo focal, secuenciado de cinco minutos. Y, al final de la sesión, coincidiendo con el tercer periodo focal, se registra otro scan del grupo. 
Para poder realizar el control de fiabilidad, se precisará de dos observadores previamente entrenados en el registro de las variables y familiarizados con los instrumentos diseñados y que permanecen durante todas las sesiones.

\subsection{Registro de datos}

Para cada periodo focal, utilizamos una tabla por categorías y tiempos de duración de cada una de ellas. Cada tabla representa a un niño, según el modelo 1 que se representa en la tabla 3:

TABLA 3: Tiempo de reacción física (modelo 1)

\begin{tabular}{|l|c|c|}
\hline Niño/a: (siglas) & Sesion. (N.) & Periodo focal. (inicio/fin) \\
\hline \multicolumn{1}{|c|}{ Categoría } & (Min./seg.) & (Min./seg.) \\
\hline Llanto & (Min./seg.) & (Min./seg.) \\
\hline Sonrisa & (Min./seg.) & (Min./seg.) \\
\hline Rostro Frío & (Min./seg.) & (Min./seg.) \\
\hline
\end{tabular}

Para cada muestreo scan, utilizamos una tabla con indicación de las variables, y de los niños, según el modelo 2 , que se representa en la tabla 4:

TABLA 4: Resultados de la Interacción (modelo 2)

\begin{tabular}{|l|l|l|}
\hline Niñola: (siglas) & Sesión: $(\mathrm{N} \cdot \mathrm{q}$ & Scan: (iniciolfin) \\
\hline \multicolumn{1}{|c|}{ Categoría } & Existe & No existe \\
\hline Alegría & $(\mathrm{s} / \mathrm{n})$ & $(\mathrm{s} / \mathrm{n})$ \\
\hline Indiferencia & $(\mathrm{s} / \mathrm{n})$ & $(\mathrm{s} / \mathrm{n})$ \\
\hline Tristeza & $(\mathrm{s} / \mathrm{n})$ & $(\mathrm{s} / \mathrm{n})$ \\
\hline
\end{tabular}

Para el análisis de los resultados, se elaborará una tabla resumen por cada niño, con indicación de los tiempos de cada una de las reacciones físicas en cada una de las seis sesiones y los totales de cada categoría; además, se realizará una tabla resumen por cada niño con indicación de las actitudes al inicio y final de cada una de las seis sesiones.

Por último, se realizarán unas tablas (inicio y final), según los modelos 3 y 4 , que se representan en las tablas 5 y 6 , en que se indicará el tipo de conducta manifestada por todos los niños en el total de sesiones, a la vez que se registrará el total de conductas manifestadas por los niños según tipología. Y otras tablas que indiquen el tiempo total de las reacciones físicas (en las seis sesiones) que manifiesta cada niño en cada uno de los periodos focales, además de indicarnos el tiempo total de las reacciones físicas para todos los niños, según el modelo 6 , que se representa en la tabla 7. 
TABLA 5: Interacción (al inicio) (modelo 3)

\begin{tabular}{|c|c|c|c|c|c|c|c|}
\hline Niño/a: (siglas) & \multicolumn{7}{|c|}{ N. de sesiones } \\
\hline Conducta emocional & 1 & 2 & 3 & 4 & 5 & 6 & TOTAL \\
\hline Alegria & & & & & & & \\
\hline Tristeza & & & & & & & \\
\hline Indiferencia & & & & & & & \\
\hline
\end{tabular}

TABLA 6: Interacción (al final) (modelo 4)

\begin{tabular}{|c|c|c|c|c|c|c|c|}
\hline Niño/a: (siglas) & \multicolumn{7}{|c|}{ No de sesiones } \\
\hline Conducta emocional & 1 & 2 & 3 & 4 & 5 & 6 & TOTAL \\
\hline Alegria & & & & & & & (N..$^{0}$ veces) \\
\hline Tristeza & & & & & & & (N..$^{\circ}$ veces) \\
\hline Indiferencia & & & & & & & (N..$^{\circ}$ veces) \\
\hline
\end{tabular}

TABLA 7: Duración de las reacciones físicas (modelo 5)

\begin{tabular}{|c|c|c|c|c|c|c|c|}
\hline Niñola: (siglas) & VIV & & & & se & & \\
\hline Conducta emocional & 1 & 2 & 3 & 4 & 5 & 6 & TOTAL \\
\hline Sonrisa & & & & & & & (Min.) \\
\hline Llanto & & & & & & & (Min.) \\
\hline Rostro Frío & & & & & & & (Min.) \\
\hline
\end{tabular}

\section{RESULTADOS}

Antes de iniciar el análisis de los resultados, es necesario indicar que éstos no han sido sometidos al análisis estadístico de concordancias, como en un principio nos planteamos, ya que la significación de las variables no ha resultado relevante a nivel cuantitativo.

Se ha realizado la validación de los instrumentos a través del sistema interjueces, ya que los dos observadores han obtenido resultados idénticos.

Una vez finalizado el trabajo de campo, pasaremos a indicar los resultados, según indicábamos anteriormente. Presentamos unas tablas resumen según el tipo de tendencia emocional manifestada por el total de niños, una para el inicio y otra para el final de las sesiones de observación (ver anexos).

Al inicio de las interacciones, la tendencia emocional más continua es la alegría, seguida de la tristeza y la indiferencia en igual medida. Y, en lo referente al final de las interacciones, la tendencia emocional 
más continua, con mucha diferencia, es la alegría, seguida de la indiferencia y la ausencia absoluta de la tristeza.

También presentamos una tabla resumen, indicando la duración en minutos de las reacciones físicas expresadas por cada uno de los niños $y$ en el total de sesiones (ver anexos).

En los resultados de las mediciones sobre las reacciones físicas han aparecido de forma más continua las reacciones físicas de rostro frío, después las sonrisas y finalmente el llanto.

Comenzamos el análisis de las actitudes mostradas por los niños en acogimiento familiar, expresadas en las sesiones de observación, y que se recogen en las tablas anexas, se resumen en las tablas 8 y 9 (ver anexos), y representadas gráficamente en los gráficos del 2 al 5 (ver anexos).

Lo referente a las reacciones físicas observadas en los niños en el total de sesiones, se registran en tablas y se resumen en la tabla 10 (ver anexos) y se expresan gráficamente en los gráficos 6 y 7 (ver anexos).

\section{CONCLUSIONES}

En la tabla 8, se resumen las tendencias emocionales de los niños mostradas al inicio. Las emociones de los niños no muestran ninguna significación global en las tres categorías observadas, ya que en 18 de las observaciones reflejan alegría, en 15 de las observaciones se refleja tristeza, y en otras 15 observaciones se refleja indiferencia.

Aunque deteniéndonos en este detalle, y si adicionamos las observaciones de estas dos últimas categorías (tristeza e indiferencia), nos encontramos con 30 observaciones con una representación emocional negativa en los niños; cuestión que nos hace inferir que la finalidad de las visitas familiares, el contacto físico entre niños y padres biológi$\cos$, no les gratifica en la mayoría de las observaciones, lo que permite acercarnos hacia la verificación de la hipótesis inicial.

Contrastando con otras investigaciones, se determina que los niños acogidos suelen perder las referencias afectivas de sus padres biológicos, reflejando con su actitud el ánimo con que los niños asumen este tipo de contactos.

Cuando nos detenemos en la observación de los niños individualmente, se hacen más significativas las diferencias. Así, los niños R.L. y A.L. muestran en todas las observaciones tristeza, ya que, prácticamente, no conocían a su madre a su padre; se les tuteló cuando contaban con meses de vida, y los únicos padres que reconocen son los acogedores. 
Por el contrario, los niños V.A. y J.M.A. muestran alegría en todas las observaciones, ya que el acogimiento pautado es de carácter temporal y la relación con los padres biológicos está facilitada por la familia acogedora, de manera que se potencia la reintegración familiar, y en el momento que se considere que las causas que motivaron la protección de los niños desaparezcan y los padres estén totalmente capacitados, podrán atender a sus hijos.

El resto de niños mostraron emociones cambiantes al iniciar las observaciones de las visitas, pasando de la «alegría» a la «indiferencia» en P.R., C.L. y A.G., y cambiante de la «tristeza» a la «indiferencia» en el niño C.M.

La observación sistemática nos permite comprobar que las emociones al inicio de las visitas se modifican en la mayoría de los niños, cuando el niño continúa con el régimen de visitas durante un proceso temporal prolongado.

Este cambio de respuesta podría deberse, entre otros, a los factores relacionados con, en primer lugar, la convivencia en las familias acogedoras y la pérdida de interés en su familia de origen y, en segundo lugar, a que los niños se acostumbran al contacto de las visitas, perdiendo afectividad como mecanismo de defensa ante la frustración.

En la tabla 9, se resumen las actitudes de los niños mostradas al final de las visitas en el total de sesiones. Así, en el gráfico 5, se observa la categoría alegría, ampliamente superior (se mostró en 36 de las observaciones realizadas al finalizar las visitas). En cuanto a las otras categorías, la indiferencia se observó en 12 ocasiones y la tristeza no se ha observado en ninguna ocasión.

La respuesta mayoritaria responde al reflejo emocional que manifiestan los niños ante la buena relación y atenciones que reciben en las familias acogedoras, en donde conviven durante el proceso de protección, y mientras se resuelven las causas que motivaron la separación de su familia biológica, si es el caso.

En otras ocasiones, se responde de manera afectivamente positiva ante la liberación de unas visitas terminadas, no muy agradables para el niño. Las emociones contrarias, de tristeza, al finalizar las visitas, reflejan una integración familiar suspendida, pero no rota, mantenida y apoyada por las familias acogedoras temporales.

Podemos inferir, pues, después de estos datos, que las visitas familiares repercuten escasamente en el mantenimiento de las relaciones paterno-filiales positivas entre los niños y sus familias biológicas.

Las reacciones físicas observadas en los niños en el total de sesiones se resumen en la tabla 10 y se representan en los gráficos 6 y 7 . 
De los resultados totales se desprende que la duración de las reacciones físicas en las observaciones se distribuye en un $51 \%$ para la categoría frialdad del rostro, seguida de la categoría sonrisa en un $32 \%$ y de la categoría llanto en un $17 \%$.

Con todos los resultados anteriores, se puede decir que posiblemente las emociones más dolorosas (tristeza) y la duración de sus reacciones físicas (llanto) más prolongadas para los niños estarán condicionadas por el tipo de relación y la duración de la convivencia entre niño y su familia de origen, por una parte, $y$, por otra parte, por las expectativas de adopción que tengan los acogedores.

De esta manera, cuanto menos tiempo ha durado la relación entre el niño y su familia de origen y más traumática sea esta relación, mayores posibilidades existirán de que el niño presente emociones de tristeza o indiferencia y mayor tiempo durarán las reacciones físicas de llanto, y viceversa.

Además, según la emoción manifestada por los niños al finalizar las visitas, tendrá una relación importante las expectativas de los acogedores con respecto a la adopción del niño, así, cuanto más tiempo permanezca el niño en la familia acogedora, más durarán las reacciones físicas de llanto de los niños al iniciar las visitas.

Completando el análisis de la interacción y de las categorías incluidas en la observación, nos centraremos en las correlaciones existentes con las reacciones físicas, sobre todo la frialdad de rostro manifestada en más de la mitad de las observaciones, determinando las emociones de indiferencia y tristeza que se observa en los niños.

Por otra parte, el llanto tiene poca significación, ya que la intervención de los educadores supervisores de las visitas familiares está especialmente volcada con los niños que presentan esta reacción física y suele remitir en poco tiempo ante su intervención.

Las sonrisas infantiles son mucho más significativas, ya que muestran la alegría del reencuentro con sus padres biológicos, y/o el retorno a los acogedores después de finalizada la visita en un ambiente no grato para el niño.

Así, podemos entender que los procesos de protección social a la infancia no son exclusivamente medidas legales o de procedimiento, llevan implícita una carga emocional importante, e influyen en los mecanismos individuales que los niños generan para poder afrontar situaciones de desintegración familiar, inadaptación social de los padres e incluso malos tratos hacia ellos o entre progenitores.

La observación sistemática de uno de estos procesos, como son las visitas familiares de los niños acogidos temporal o indefinidamente en familias seleccionadas con sus familias biológicas, nos permite preci- 
samente reconocer las incidencias que concurren en estas situaciones de interacción.

Las reacciones que manifiestan los niños revelan inferencias respecto de los vínculos parentales de las relaciones con las familias acogedoras y de la conveniencia o no de una reintegración familiar con garantías para el niño.

A modo de pronóstico, diremos que las visitas entre los niños acogidos judicialmente y sus familias de origen supondrán un riesgo para la integración familiar del niño, siempre que las emociones mostradas al inicio y al final de las visitas supongan un perjuicio para la emotividad del niño, y la duración de las reacciones físicas de llanto y frialdad en el rostro será mayor en función del tiempo de permanencia en acogida familiar judicial.

En este sentido, el derecho a la realización de estas visitas y su efectividad deberá ser analizado desde unas perspectivas relacionadas con las causas que crearon esta situación y las expectativas de los padres naturales ante las mismas.

Por lo tanto, la consideración legal de este régimen de visitas debe plantearse desde la influencia que en otros aspectos genere en los niños.

Aspectos psicológicos que están presentes en la interacción de los dos ámbitos, el natural y el de acogida, que se muestra como compleja y en ocasiones conflictiva, antes de resolver la demanda de visitas por parte de la familia biológica, deberán tenerse en cuenta las variables que consideramos en este estudio, además de las que apuntamos en esta conclusión final y, sobre todo, tener en cuenta que, por encima de los derechos y solicitudes de las familias de origen, están los derechos e intereses de los niños en situación de acogimiento familiar judicial, medida que, por cierto, ya lleva implícita, per se, un grave deterioro de la estructura familiar de origen.

Por último, quisiéramos llamar la atención hacia la reflexión del porqué de la reacción de un niño ante la inminente presencia de sus padres biológicos, porque es muy reveladora y útil para quienes han de tomar las decisiones pertinentes sobre la idoneidad de continuar promoviendo o suspendiendo este tipo de interacción. En ocasiones, las visitas pueden ser imperativos legales, pero deben proponerse teniendo en cuenta las posibles incidencias positivas o negativas en la situación emocional del niño. 


\section{BIBLIOGRAFIA}

ANGUERA, M ${ }^{\mathrm{a}} \mathrm{T}$.: Metodología de la observación en las ciencias humanas. Cátedra. Madrid, 1992.

BOWLBY, J.: El vínculo afectivo. Paidós. Buenos Aires, 1979.

BOWLBY, J.: La separación afectiva. Paidós. Buenos Aires, 1979.

BUENO ABAD, J.R.: "Retos y desafíos para la intervención psicosocial". En Informació Psicológica, V época $\mathrm{n}^{\circ}$ 69. Col.legi Oticial de Psicólegs del País Valencià. Valencia, 1999. Pp: 42-49.

BUENO ABAD, J.R.: Representationes Sociales de l'inadaptation. Memoir de l'École des Hautes Études Sociales. París, 1998.

CAPLAN, G.: Support systems and community mental health. Basic Books. New York, 1974.

CLEMENTE, M.: Psicología Social. Métodos y técnicas de Investigación. Eudema Universidad. Madrid, 1992.

DELGADO, J.M. Y GUTIÉRREZ, J. (Coord.): Métodos y técnicas cualitativas de investigación en Ciencias Sociales. Síntesis Psicológica. Madrid, 1995.

GARCÍA FERRANDO, M., IBÁÑEZ, J., ALVIRA, F.: El análisis de la realidad social. Métodos y técnicas de investigación. Alianza Universidad. Madrid, 1993.

GERGEN, K.: Psychologie Sociale. Vigot. Quebec, 1992.

GUASCH, O.: Observación participante. CIS. Madrid, 1997.

L'ECUYER, R.: Méthode GPS et concept de soi. Presses de l'Université de Quebec. Quebec, 1990.

MAYNTZ, R., HOLM, K., HÜBNER, P.: Introducción a los métodos de sociología empírica. Alianza Universidad. Madrid, 1988.

PÉREZ COSÍN, J.V.: "Quiestions sobre adopció internacional". En Codi-46. I época n². Col.legi Oficial de Diplomats en Treball Social i Assistents Socials de València. Massamagrell (Valencia), 1993. Pp: 24-25.

RIPOL-MILLET, A. y RUBIOL, G.: L'acolliment familiar. Frontissa. Barcelona, 1988.

RIPOL-MILLET, A. y RUBIOL, G.: El acogimiento familiar. Ministerio de Asuntos Sociales. Madrid, 1990.

RUIZ OLABUÉNAGA, J.I.: Metodología de la investigación cualitativa. Universidad de Deusto. Bilbao, 1996.

SELLTIZ, C., WRIGHTSMAN, L., COOK, S.: Métodos de investigación en las relaciones sociales. Alianza. Madrid, 1980.

SIERRA, R.: Técnicas de investigación social. Teoría y ejercicios. Paraninfo. Madrid, 1987. 
TAYLOR, S.J. y BOGDAN, R.: Introducción a los métodos cualitativos de investigación. Paidós. Barcelona, 1998.

VALLÉS, M.: Técnicas cualitativas de investigación social. Reflexión metodológica y práctica profesional. Síntesis Sociológica. Madrid, 1997.

\section{ANEXOS}

TABLA 8: Resumen de las tendencias emocionales de los niños (al inicio)

\begin{tabular}{|l|c|c|c|}
\hline NIÑOS/AS (siglas) & AUEGRIA & IRISTEZA & INDIFERENCIA \\
\hline P.R. & 2 & 0 & 4 \\
\hline R.L. & 0 & 6 & 0 \\
\hline A.L. & 0 & 6 & 0 \\
\hline V.A. & 6 & 0 & 0 \\
\hline J.M.A. & 6 & 0 & 0 \\
\hline C.L. & 2 & 0 & 4 \\
\hline C.M. & 0 & 3 & 3 \\
\hline A.G. & 2 & 0 & 4 \\
\hline . TOTAUES & $\mathbf{1 8}$ & $\mathbf{1 5}$ & $\mathbf{1 5}$ \\
\hline
\end{tabular}

Fuente y elaboración propias.

GRÁFICO 2

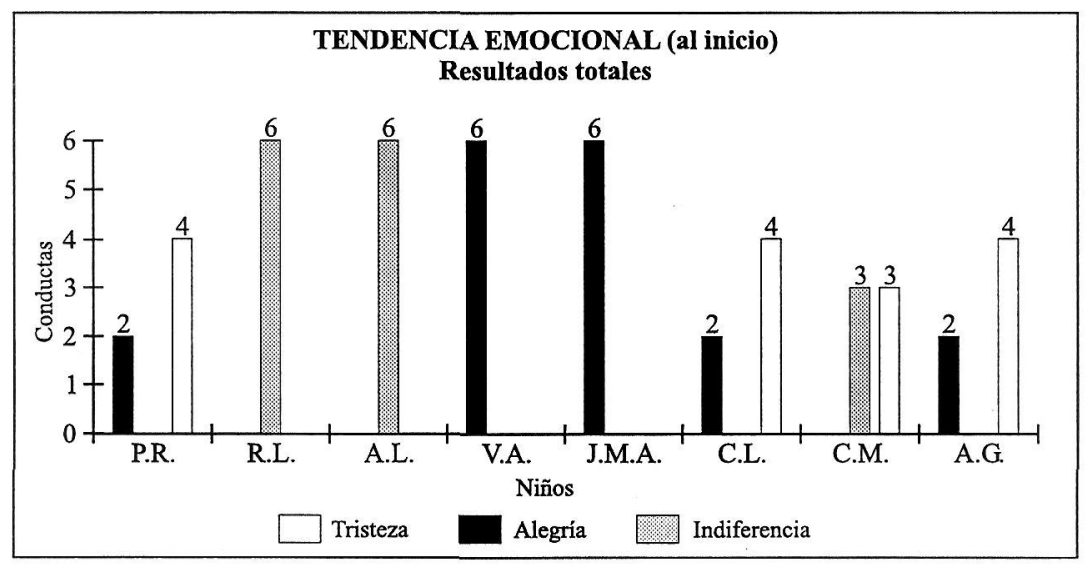

Elaboración propia. 


\section{GRÁFICO 3}

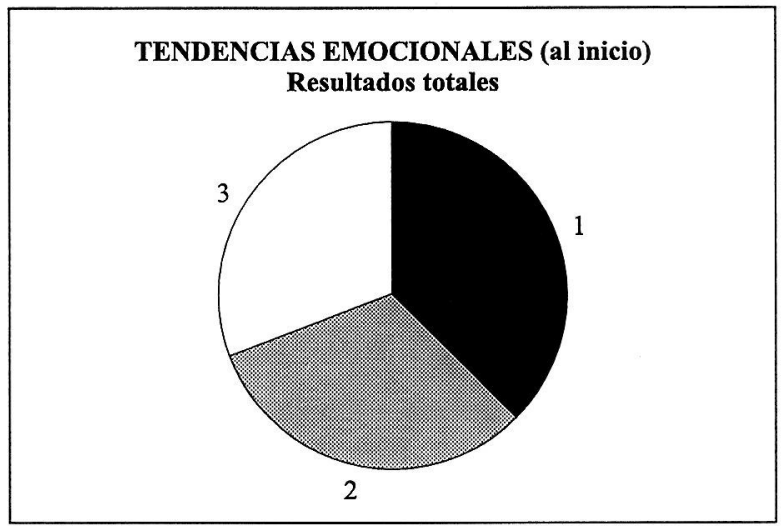

Elaboración propia.

TABLA 9: Resumen de las tendencias emocionales de los niños (al final)

\begin{tabular}{|c|c|c|c|}
\hline NINOS/AS (siglas) & ALEGRIA & TRISTEZA A & INDIFERENCIA \\
\hline P.R. & 6 & 0 & 0 \\
\hline R.L. & 0 & 0 & 6 \\
\hline A.L. & 0 & 0 & 6 \\
\hline V.A. & 6 & 0 & 0 \\
\hline J.M.A. & 6 & 0 & 0 \\
\hline C.L. & 6 & 0 & 0 \\
\hline C.M. & 6 & 0 & 0 \\
\hline A.G. & 6 & 0 & 0 \\
\hline TOTALES & 36 & 0 & 12 \\
\hline
\end{tabular}

Fuente y elaboración propias. 


\section{GRÁFICO 4}

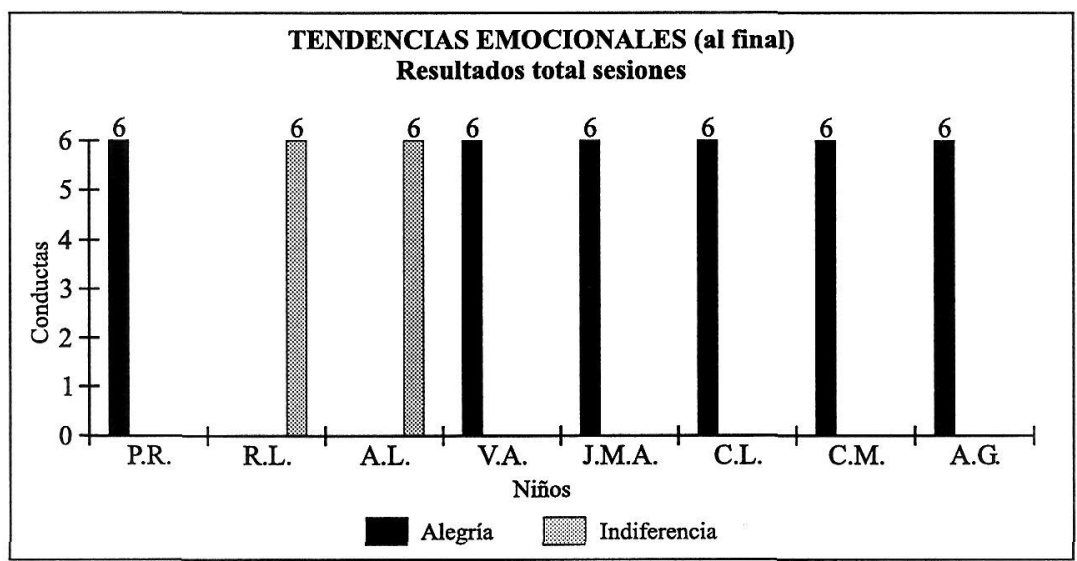

Elaboración propia.

GRÁFICO 5

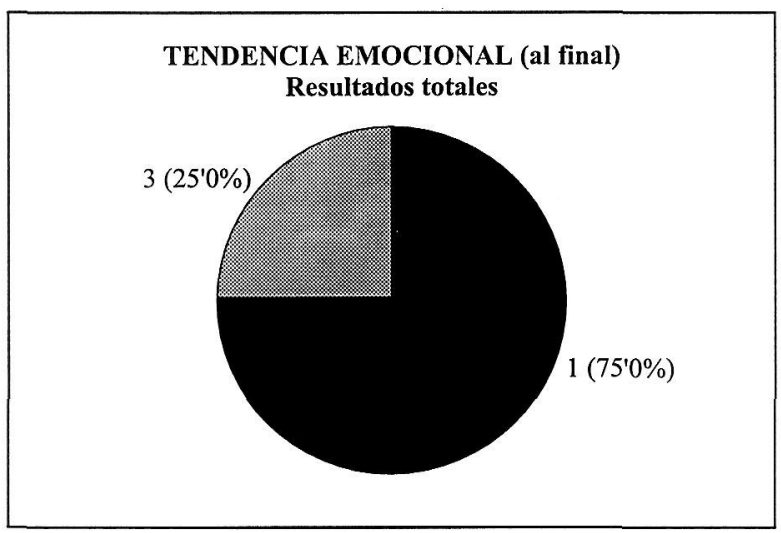

Elaboración propia. 
TABLA 10: Resumen de la duración de las reacciones físicas (Min.)

\begin{tabular}{|c|c|c|c|}
\hline NINOS/AS (siglas) & SONRISA & I.ANMTO & ROSTRO FRIÓO \\
\hline P.R. & 47 & 0 & 43 \\
\hline R.L. & 0 & 48 & 42 \\
\hline A.L. & 0 & 42 & 48 \\
\hline V.A. & 72 & 0 & 18 \\
\hline J.M.A. & 47 & 11 & 32 \\
\hline C.L. & 25 & 0 & 65 \\
\hline C.M. & 0 & 22 & 67 \\
\hline A.G. & 42 & 0 & 48 \\
\hline TOTALES & 233 & 123 & 363 \\
\hline
\end{tabular}

Fuente y elaboración propias.

GRÁFICO 6

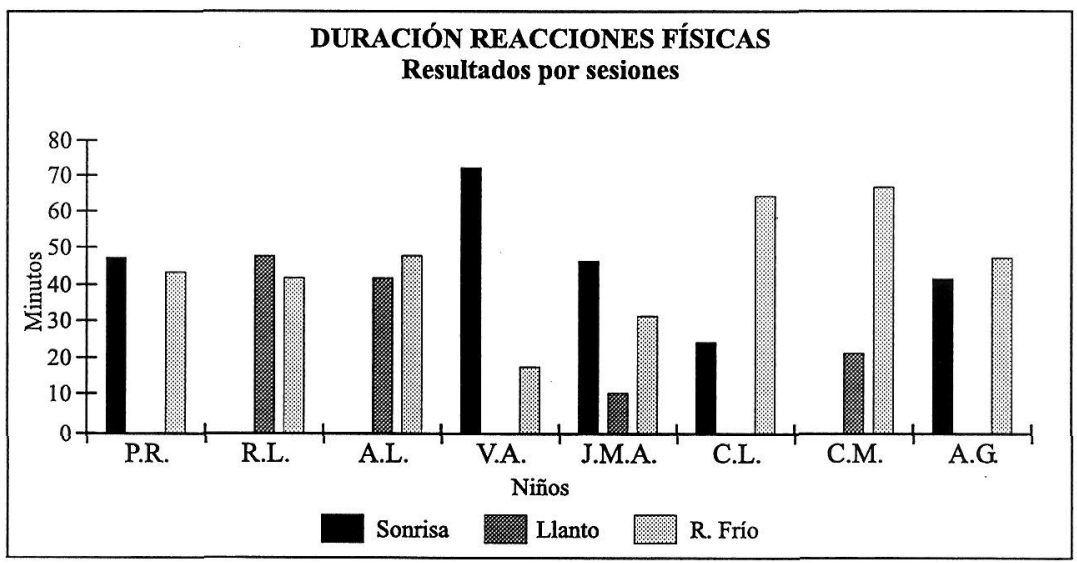

Elaboración propia. 
GRÁFICO 7

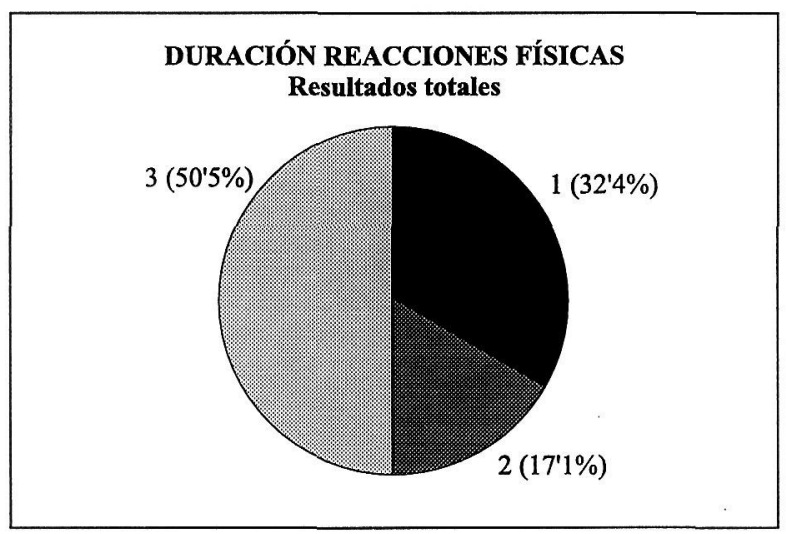

Elaboración propia. 